\title{
Sehr verehrter, lieber Herr Dr. Stöcklin
}

Wenn an Ihrem 60. Geburtstage tausende dankbare Patienten ihres jetzigen oder einstigen Arztes gedenken, so geschieht es mit ganz besonderer Wärme aus dem Kreise Ihrer früheren Assistenten. Als Heil- und nicht als Lehrbedürftige haben die meisten von uns Sie zunächst kennengelernt. Wir erlebten dabei Ihr menschliches Interesse, mit dem Sie die Tuberkulose als AUgemeinkrankheit erfaßten, wir bewunderten Ihre exakten und klaren Richtlinien in der Erkennung und Behandlung der Tuberkulose, Ihre Kunst der Diagnostik und Ihre vorbildliche Organisation des Heilstättenbetriebes. So wurden wir über die Heilung der eigenen Krankheit hinaus durch das Vorbild Ihres medizinischen und menschlich sozialen Wirkens zur Phthisiologie hingeführt und lernten sie als Lebensaufgabe bejahen.

Ihr persönliches Gepräge hat der Thurg.-Schaffh. Heilstätte, der Sie seit der Gründung vorstehen, zu ihrem hohen Ruf verholfen; sie ist heute anerkanntes und viel studiertes Muster einer Heilstätte überhaupt.

Neben der Lösung der vielfältigen praktischen Arbeiten haben Sie stets das wissenschaftliche Problem der Tuberkulose in seiner ganzen Be-deutung gesehen, haben durch Ihre eigenen Forschungen und vor allem auch durch die aus Ihrem Geiste inspirierten wissenschafthchen Arbeiten Wesentliches zur Tuberkuloseforschung beigetragen. Damit wurden Sie in der Schweiz zum Begründer einer eigentlichen Phthisiologenschule, aus der zahlreiche, heute teilweise an leitender Stelle tätige Lungenärzte hervoi-gegangen sind. Diese legen in ihren Forschungen und in der täglichen, an Ihrem Vorbild sich messenden Arbeit Zeugnis ab für Ihr Wirken und Wollen. Darüber hinaus wird das wesentlich durch Ihr Verdienst geschaffene neue TuberkuloseForschungsinstitut in Davos die Ausstrahkmgen Ihres Bestrebens verstärken.

Die Beiträge in dieser Festnummer der „Schweizerischen Zeitschrift für Tuberkulose”, die wir Ihnen, sehr verehrter Herr Dr. Stöcklin, widmen, möchten ein bescheidener Ausdruck unserer Gefühle der Dankbarkeit und in ihrer Vielfalt ein Spiegel Ihres eigenen so fruchtbaren Lebenswerkes sein, für dessen Weiterführung und Erfüllung wir Ihnen die herzlichsten Wunsche überbringen.

Jakob Steiger Albert Wernh-Haessig

Auch die Redaktion der Zeitschrift entbietet Herrn Dr. Stöcklin, Chefarzt der Thurgauischen Heilstätte in Davos, die herzlichsten Gl-ück-wünsche zu seinem 60. Geburtstage und dankt ihm für die große Arbeit, die er für die Erforschung und Behandlung der Tuberkulose geleistet hat. Im Namen des Redaktionskomitees

E. Bachmann E. Uehlinger

INDEX

Haefliger, Zur Entstehung der Lungentuberkulose

Landolt, Verschwartende Pleuritis exsudativa bei Bronchustuberkulose . . 238

Mauderlí, Uber die Entstehung unspezifisch-entzündlicher Hilusveränderungen 245

Mordasiní, Beitrag zur niodernen Behandlung der tuberkulösen Meningitis

beim Erwachsenen 
Ott, Die Tuberkulose-Mortalität und -Morbidität inihrenBeziehungen zu den Fortschritten in der Tuberkulosebekämpfung und den sozialen Ein-

richtungenim Kanton Solothurn 1900-1950

266

Ott und Tanner, Uber unsere Resultate mit Streptomycin und PAS in der

Behandlung der Urogenitaltuberkulose 291

Schachenmann, Uber den Verlauf primärer Hauttuberkulosen

302

Steiger, Siliko-Tuberkulose

310

Steíner, Exstirpation des Ganglion stellatum bei Asthma bronchiale ....

Suter und Iselin, Zur Frage der Entstehung der Lungenphthise des Er

wachsenen aus perforierenden Hiluslymphknoten

341

Wacker, Avantages de la tomographie latérale du thorax

349

Wehrlín, Zur Differentialdiagnose der Meningitis tuberculosa

Wernlí-Haessig, Team-Work in der Behandlung der Lungenkranken ....

Libri

368 